\title{
On the convergence of cluster expansions for polymer gases
}

\author{
Rodrigo Bissacot $^{1,2}$, Roberto Fernández ${ }^{2,3}$, Aldo Procacci ${ }^{1}$ \\ ${ }^{1}$ Dep. Matemática-ICEx, UFMG, CP 702 Belo Horizonte - MG, 30161-970 Brazil \\ ${ }^{2}$ Labo. de Maths Raphael Salem, Université de Rouen, 76801, France \\ ${ }^{3}$ Department of Mathematics, Utrecht University, P.O. Box 800103508 TA Utrecht \\ emails: rodrigo.bissacot@gmail.com; Roberto.Fernandez@univ-rouen.fr; R.Fernandez1@uu.nl; \\ aldo@mat.ufmg.br
}

\begin{abstract}
We compare the different convergence criteria available for cluster expansions of polymer gases subjected to hard-core exclusions, with emphasis on polymers defined as finite subsets of a countable set (e.g. contour expansions and more generally high- and low-temperature expansions). In order of increasing strength, these criteria are: (i) Dobrushin criterion, obtained by a simple inductive argument; (ii) Gruber-Kunz criterion obtained through the use of Kirkwood-Salzburg equations, and (iii) a criterion obtained by two of us via a direct combinatorial handling of the terms of the expansion. We show that for subset polymers our sharper criterion can be proven both by a suitable adaptation of Dobrushin inductive argument and by an alternative - in fact, more elementary - handling of the KirkwoodSalzburg equations. In addition we show that for general abstract polymers this alternative treatment leads to the same convergence region as the inductive Dobrushin argument and, furthermore, to a systematic way to improve bounds on correlations.
\end{abstract}

\section{Introduction}

The most basic and frequent applications of cluster expansions deal with (log of) partition functions of random geometric objects subjected only to hard-core exclusions. The relevant mathematical structure was first formalized by Gruber and Kunz [13 for the case of objects defined by subsets of a countable set. They called these objects polymers. A decade later Kotecký and Preiss [14] introduced more general families of objects that are not necessarily subsets of an underlying set and whose "hard-core" interaction is defined by an incompatibility relation. This more general objects will be called abstract polymers in the sequel, while subset polymers will be those introduced by Gruber and Kunz.

In their seminal paper, Gruber and Kunz used Kirkwood-Salzburg equations to determine convergence radii. This GK approach, however, did not become popular and instead practitioners turned to bounds obtained by first showing that cancellations yield a majorizing expansion in terms of tree diagrams. The convergence condition is proved by inductively summing the "leaves" of the expansion. The genesis of this approach is attributed to Cammarota [6], and the canonical reference is the excellent review by Brydges [4] (see also [5], 21], 22]). In contrast, Kotecký and Preiss introduced an inductive argument that does not make any reference to the actual expression of the series. This argument, helped by a refinment by Dobrushin [7], became the argument-of-choice in further developments [16, 3, 15, 26, 27, 24]. There are good reasons 
for this: the inductive argument leads to notoriously simpler convergence proofs and stronger results than the more laborious tree sums (see [8] for a remarkable overview of consequences of these results). In particular it leads quite naturally to a bound on correlationns and "pinned" free energies. But there is a downside: the argument works "too well". While Dobrushin's condition is perfectly designed to survive the inductive step, the method contains no hint on how to obtain further improvements.

To break this impasse, in [9] we went back to basics. We took a hard look at the cluster expansion in full and studied it avoiding inequalities as much as possible. The breakthrough came from a seldomly remembered paper by Oliver Penrose [17] where the series is written in terms of a tree-grap identity involving trees determined by compatibility constraints. This yields a series whose partial sums can be generated as successive applications of a fixed transformation. Convergence criteria are then found by suitably bounding this transformation. In this way we were able to improve Dobrushin criterion and, furthermore, explain the loss of precision of preexisting criteria as incomplete accounting of Penrose constraints. Thus, on the positive side we managed to clarify the role of different approximations and to obtain stronger results, while leaving in the background a tree expansion that could be used for further refinments (see e.g. [19, 20, 18]). On the negative side, however, our method lacks the elegance and economy of the inductive Kotecký-Preiss-Dobrushin (KPD) approach. We have already exploited the positive side to improve results in a number of well-studied applications [2, 10, 11, 12]. The present paper addresses the negative side.

In [9] we also applied our new criterion to subset polymers, resorting to some simplifications to obtain a expression suitable for computations. We were surprised to find out that the "new" resulting criterion while being clearly better than Dobrushin's is in fact identical to the long forgotten Gruber-Kunz bound. There is only a small difference in our favor: the GK bound involve a strict inequality while in ours the inequality is not strict. That is why we call our bound the extended GK criterion. This state of affairs motivated a number of questions that sufaced repeatedly in discussions with our colleagues and motivated the $\mathrm{PhD}$ thesis of one of us [1]:

(Q1) Does the use of Kirkwood-Salzburg equations à la Gruber-Kunz yield better bounds than the inductive KPD approach?

(Q2) Can Kirkwood-Salzburg equations lead to an alternative proof of our new criterion?

(Q3) More ambitiously: Is there an inductive proof — à la Dobrushin — of our new criterion?

In this paper we answer these questions for the case of subset polymers, and provide partial answers for general abstract polymers. In more detail we prove the following:

(A1) For subset polymers:

(A1.1) An alternative handling of the Kirkwood-Salzburg equations proves the extended GK criterion. Thus, Kirkwood-Salzburg equations can indeed be used to prove our new convergence criterion and bounds on correlations for these polymers. The alternative handling consists in replacing the Banach-space fixed-point theorem of the original approach by a more elementary argument on convergence of series with positive terms. The extended criterion follows from writing this expansion as a limit of iterations of a fixed transformation, following an idea from [9]. 
(A1.2) Suitably adapted, an inductive KPD-approach can also be used to prove the extended GK criterion. Therefore, for subset polymers the three approaches - GK, inductive KPD and ours - are equivalent.

(A2) Likewise, the GK approach with modified handling of the Kirkwood-Salzburg equations is equivalent to the inductive KPD approach for general abstract polymers.

In the general setup of abstract polymers, we are at present unable to prove our improved criterion using either of these two equivalent approaches. The obstacle, explicitly seen in our treatment below, is the use of factorized majorizing weights inherent to both the GK and the KPD approaches.

The paper is organized so to be reasonably self-contained. In Section 2 we review the general definition of abstract and subset polymer gases and we present and compare the different convergence criteria. In Section 3 we show how to obtain the (extended) Gruber-Kunz criterion through an inductive argument. We adapt a simple argument by Miracle-Solé [15] that relies on the alternating-sign property of the truncated coefficients. In Section 4 we review Gruber-Kunz setting of Kirkwood-Salzburg equations for subset polymers and prove the extended GK bound by introducing the alternative treatment of these equations. In Section 5 we show how Dobrushin criterion can also be obtained from Kirkwood-Salzburg equations in the general abstract case. We conclude with some final comments and suggestions.

\section{Polymer gases and convergence criteria}

First, some general notation. For a set $U$ we denote $|U|$ its cardinality and $\mathbb{1}_{\{U\}}$ its indicator function.

\subsection{The abstract polymer gas}

An abstract polymer system is a triple $(\mathcal{P}, \mathcal{R}, \boldsymbol{z})$ where

- $\mathcal{P}$ is a countable set, whose elements $\gamma$ we call polymers, following Gruber and Kunz.

- $\mathcal{R} \subset \mathcal{P} \times \mathcal{P}$ is a symmetric and reflexive relation. When $\left(\gamma, \gamma^{\prime}\right) \in \mathcal{R}$, we write $\gamma \neq \gamma^{\prime}$ and say that $\gamma$ and $\gamma^{\prime}$ are incompatible. Conversely, if $\left(\gamma, \gamma^{\prime}\right) \notin \mathcal{R}$ we say that the polymers $\gamma$ and $\gamma^{\prime}$ are compatible and we write $\gamma \sim \gamma^{\prime}$. Note that the assumption that $\mathcal{R}$ is reflexive implies that $\gamma \not \gamma$ for all $\gamma \in \mathcal{P}$.

- $z: \mathcal{P} \rightarrow \mathbb{C}: \gamma \mapsto z_{\gamma}$ is the activity function. The number $z_{\gamma}$ is called the activity of the polymer $\gamma$.

The corresponding polymer gas is defined by complex-valued measures. For each finite family of polymers $\Lambda \subset \mathcal{P}$ a measure is defined assigning, to each polymer configuration $\left\{\gamma_{1}, \ldots, \gamma_{n}\right\} \subset$ $\Lambda, n \geq 0$, the weight

proba

$$
\mathbb{P}_{\Lambda}\left(\boldsymbol{z}, \gamma_{1}, \ldots, \gamma_{n}\right)=\frac{1}{\Xi_{\Lambda}(\boldsymbol{z})} z_{\gamma_{1}} z_{\gamma_{2}} \ldots z_{\gamma_{n}} \prod_{1 \leq i<j \leq n} \mathbb{1}_{\left\{\gamma_{i} \sim \gamma_{j}\right\}}
$$


with the convention $\left\{\gamma_{1}, \ldots, \gamma_{n}\right\}=\emptyset$ when $n=0$ and $\mathbb{P}_{\Lambda}(\boldsymbol{z}, \emptyset)=1 / \Xi_{\Lambda}(\boldsymbol{z})$. Here

$$
\begin{aligned}
\Xi_{\Lambda}(\boldsymbol{z}) & =1+\sum_{n \geq 1} \frac{1}{n !} \sum_{\left(\gamma_{1}, \ldots, \gamma_{n}\right) \in \Lambda^{n}} z_{\gamma_{1}} z_{\gamma_{2}} \ldots z_{\gamma_{n}} \prod_{1 \leq i<j \leq n} \mathbb{1}_{\left\{\gamma_{i} \sim \gamma_{j}\right\}} \\
& =1+\sum_{n \geq 1} \sum_{\left\{\gamma_{1}, \ldots, \gamma_{n}\right\} \in \Lambda} z_{\gamma_{1}} z_{\gamma_{2}} \ldots z_{\gamma_{n}} \prod_{1 \leq i<j \leq n} \mathbb{1}_{\left\{\gamma_{i} \sim \gamma_{j}\right\}}
\end{aligned}
$$

normalizes $\mathbb{P}_{\Lambda}(\boldsymbol{z}, \Lambda)=1$. The equality betwwen the first and second lines follows from the fact that, in the presence of compatibility requirements, only $n$-t-uples with different components contribute. Restricted to positive fugacities, $\left\{z_{\gamma} \geq 0\right\}_{\gamma \in \mathcal{P}}$, the measure $\mathbb{P}_{\Lambda}$ is a probability measure on the space of subsets of $\Lambda$ and $\mathbb{P}_{\Lambda}\left(\boldsymbol{z}, \gamma_{1}, \ldots, \gamma_{n}\right)$ is interpreted as the probability of observing exactly polymers $\gamma_{1}, \ldots, \gamma_{n}$ out of the family $\Lambda$. In general, weights are allowed to be complex to settle analyticity questions. The normalization constant $\Xi_{\Lambda}(\boldsymbol{z})$ is interpreted as the grand-canonical partition function of the family $\Lambda$. This partition function $\Xi_{\Lambda}(\boldsymbol{z})$ is the key function from which all "physical quantities" of the system can be derived. These quantities include the "pressure" of the system

$$
P_{\Lambda}(\boldsymbol{z})=\frac{1}{|\Lambda|} \log \Xi_{\Lambda}(\boldsymbol{z})
$$

and the correlations

$$
\phi_{\Lambda}\left(\boldsymbol{z}, \gamma_{1}, \ldots, \gamma_{p}\right)=\left[z_{\gamma_{1}} \ldots z_{\gamma_{p}} \prod_{1 \leq i<j \leq p} \mathbb{1}_{\left\{\gamma_{i} \sim \gamma_{j}\right\}}\right] \frac{\Xi_{\Lambda \backslash \Gamma\left(\cup_{i=1}^{p} \gamma_{i}\right)}(\boldsymbol{z})}{\Xi_{\Lambda}(\boldsymbol{z})} .
$$

assuming $\left\{\gamma_{1}, \ldots, \gamma_{p}\right\} \subset \Lambda$ and denoting, for any finite family of polymers $X$,

$$
\Gamma(X)=\left\{\gamma \in \mathcal{P}: \exists \gamma^{\prime} \in X \text { such that } \gamma \not \gamma^{\prime}\right\}
$$

- the neighborhood of $X$. We see from (2.4) that all is decided by the ratios

$$
\bar{\phi}_{\Lambda}(\boldsymbol{z}, X)=\frac{\Xi_{\Lambda \backslash X}(\boldsymbol{z})}{\Xi_{\Lambda}(\boldsymbol{z})} \quad X \subset \Lambda
$$

which Gruber and Kunz baptized the reduced correlations.

The main issues of the theory are the existence and analyticity with respect to fugacities of the $\Lambda \rightarrow \mathcal{P}$ limits of the pressure and correlation functions. The most detailed way of answering these questions is by writing $\log \Xi_{\Lambda}(\boldsymbol{z})$ as a formal power series in the fugacities. This series, historically called Mayer series, takes the form

$$
\log \Xi_{\Lambda}(\boldsymbol{z})=\sum_{n=1}^{\infty} \frac{1}{n !} \sum_{\left(\gamma_{1}, \ldots, \gamma_{n}\right) \in \Lambda^{n}} \phi^{T}\left(\gamma_{1}, \ldots, \gamma_{n}\right) z_{\gamma_{1}} z_{\gamma_{2}} \ldots z_{\gamma_{n}}
$$

where the truncated coefficients $\phi^{T}\left(\gamma_{1}, \ldots, \gamma_{n}\right)$ depend only on the compatibility graph of the argument. This graph has vertex set $\{1,2, \ldots, n\}$ and edge set $\left\{\{i, j\} \subset\{1,2, \ldots, n\}: \gamma_{i} \nsim \gamma_{j}\right\}$. [Warning! The truncated coefficients do not require compatibility of their arguments, hence the analogous of the second line of (2.2) is not valid for (2.7).] The methods discussed in this paper 
do not make use of the actual expression of the truncated coefficients (which can be found, for instance, in [6, 4, 5, 21, 22]). Below we only need the alternating-sign property

sign

$$
\left|\phi^{T}\left(\gamma_{1}, \ldots, \gamma_{n}\right)\right|=(-1)^{n-1} \phi^{T}\left(\gamma_{1}, \ldots, \gamma_{n}\right),
$$

which can be easily derived from the Penrose identity [17] (see e.g. 9] and [24]) or by a simple induction argument [15].

In view of $(2.6)$ it is natural to focus on the differences

$$
\begin{aligned}
\Theta_{\gamma}^{\Lambda}(\boldsymbol{z}) & =\log \Xi_{\Lambda}(\boldsymbol{z})-\log \Xi_{\Lambda \backslash\{\gamma\}}(\boldsymbol{z}) \\
& =\sum_{n=1}^{\infty} \frac{1}{n !} \sum_{\substack{\left(\gamma_{1}, \ldots, \gamma_{n}\right) \in \Lambda^{n} \\
\exists i: \gamma_{i}=\gamma}} \phi^{T}\left(\gamma_{1}, \ldots, \gamma_{n}\right) z_{\gamma_{1}} \ldots z_{\gamma_{n}} .
\end{aligned}
$$

From them we can reconstruct

reconst

$$
\log \Xi_{\Lambda}(\boldsymbol{z})=\sum_{\gamma \in \Lambda} \Theta_{\gamma}^{\Lambda}(\boldsymbol{z})
$$

and, for $\left\{\gamma_{1}, \ldots, \gamma_{p}\right\} \subset \Lambda$,

recons $-x$

$$
\bar{\phi}_{\Lambda}\left(\boldsymbol{z},\left\{\gamma_{1}, \ldots, \gamma_{p}\right\}\right)=\exp \left(-\sum_{i=1}^{p} \Theta_{\gamma_{i}}^{\Lambda \backslash\left\{\gamma_{i+1}, \ldots, \gamma_{p}\right\}}(\boldsymbol{z})\right) .
$$

In order to state the theorems of the next section, we will also need to consider another series, directly related to $\Theta_{\gamma}^{\Lambda}(\boldsymbol{z})$, namely

$$
\Pi_{\gamma}^{\Lambda}(\boldsymbol{z})=\frac{\partial}{\partial z_{\gamma_{0}}} \log \Xi_{\Lambda}\left(z_{\Lambda}\right)
$$

$P i$

$$
=\sum_{n=0}^{\infty} \frac{1}{n !} \sum_{\left(\gamma_{1}, \gamma_{2}, \ldots, \gamma_{n}\right) \in \Lambda^{n}} \phi^{T}\left(\gamma, \gamma_{1}, \ldots, \gamma_{n}\right) z_{\gamma_{1}} \ldots z_{\gamma_{n}}
$$

Series (2.7), (2.9) and (2.12) are examples of cluster expansions; we will focus on the last two. Analyticity results are obtained from it on the basis of the observation that, for a family of positive numbers $\left\{\rho_{\gamma}\right\}_{\gamma \in \mathcal{P}}$, the positive-term series

$6 a b s$

$$
\begin{gathered}
|\Theta|_{\gamma}^{\Lambda}(\boldsymbol{\rho})=\sum_{n=1}^{\infty} \frac{1}{n !} \sum_{\substack{\left(\gamma_{1}, \ldots, \gamma_{n}\right) \in \Lambda^{n} \\
\exists i: \gamma_{i}=\gamma}}\left|\phi^{T}\left(\gamma_{1}, \ldots, \gamma_{n}\right)\right| \rho_{\gamma_{1}} \cdots \rho_{\gamma_{n}} \\
|\Pi|_{\gamma}^{\Lambda}(\boldsymbol{\rho})=\sum_{n=0}^{\infty} \frac{1}{n !} \sum_{\substack{\left(\gamma_{1}, \gamma_{2}, \ldots, \gamma_{n}\right) \in \Lambda^{n} \\
\mid}}\left|\phi^{T}\left(\gamma, \gamma_{1}, \ldots, \gamma_{n}\right)\right| \rho_{\gamma_{1}} \ldots \rho_{\gamma_{n}}
\end{gathered}
$$

dominates (2.9) term-by-term for $\left|z_{\gamma}\right| \leq \rho_{\Lambda}$. Therefore, convergence of this last series implies the absolute and uniform convergence of (2.9) and (2.12) in the polydisc

$$
\mathcal{D}_{\boldsymbol{\rho}}=\left\{\boldsymbol{z}:\left|z_{\gamma}\right| \leq \rho_{\gamma}\right\}
$$


and its analyticity (with respect to the fugacities) in its interior. In fact, the alternating-sign property (2.8) implies that

minus

minusa

$$
\begin{gathered}
|\Theta|_{\gamma}^{\Lambda}(\boldsymbol{\rho})=-\Theta_{\gamma}^{\Lambda}(\boldsymbol{z}=-\boldsymbol{\rho}) . \\
|\Pi|_{\gamma}^{\Lambda}(\boldsymbol{\rho})=\Pi_{\gamma}^{\Lambda}(\boldsymbol{z}=-\boldsymbol{\rho}) .
\end{gathered}
$$

Thus, for finite $\Lambda$, the convergence of the series (2.13) and (2.14) is a necessary and sufficient condition for the convergence of the cluster expansion (2.9) and (2.12) in the polydisc $\mathcal{D}_{\boldsymbol{\rho}}$. By (2.10), (2.11) and (2.12) these properties are inherited by the correlations and the pressure. To extend these existence and analyticity results to the limit $\Lambda \rightarrow \mathcal{P}$ the strategy is to prove that the convergence of (2.13) happens for $\Lambda$-independent values of $\rho_{\Lambda}$.

\subsection{Convergence criteria for abstract polymer gases}

We focus on two criteria. First, Dobrushin's:

Theorem 2.1 [7] Let $\boldsymbol{\mu}=\left\{\mu_{\gamma}\right\}_{\gamma \in \mathcal{P}}$ and $\boldsymbol{\rho}=\left\{\rho_{\gamma}\right\}_{\gamma \in \mathcal{P}}$ be collections of nonnegative numbers such that

do

$b d o$

do.bo

with

$$
\rho_{\gamma} \varphi_{\gamma}^{\mathrm{D}}(\boldsymbol{\mu}) \leq \mu_{\gamma} \quad \forall \gamma \in \mathcal{P}
$$

then the series $|\Theta|_{\gamma}^{\Lambda}(\boldsymbol{\rho}),|\Pi|_{\gamma}^{\Lambda}(\boldsymbol{\rho})$ defined in (2.13), (2.14) are convergent and furthermore

$$
\begin{gathered}
|\Pi|_{\gamma}^{\Lambda}(\boldsymbol{\rho}) \leq \varphi_{\gamma}^{\mathrm{D}}(\boldsymbol{\mu}) \\
|\Theta|_{\gamma}^{\Lambda}(\boldsymbol{\rho}) \leq \log \left(1+\mu_{\gamma}\right) .
\end{gathered}
$$

We denote $\Gamma(\gamma) \equiv \Gamma(\{\gamma\})$ the neighborhood of $\gamma$, namely $\Gamma(\gamma)=\{\tilde{\gamma} \in \mathcal{P}: \tilde{\gamma} \not \gamma\}$. Here is the second criterion, which improves Dobrushin's.

Theorem 2.2 [9] Let $\boldsymbol{\mu}=\left\{\mu_{\gamma}\right\}_{\gamma \in \mathcal{P}}$ and $\boldsymbol{\rho}=\left\{\rho_{\gamma}\right\}_{\gamma \in \mathcal{P}}$ be collections of nonnegative numbers such that

$f p$

$$
\rho_{\gamma} \varphi_{\gamma}^{\mathrm{FP}}(\boldsymbol{\mu}) \leq \mu_{\gamma} \quad \forall \gamma \in \mathcal{P}
$$

with

$$
\varphi_{\gamma}^{\mathrm{FP}}(\boldsymbol{\mu})=\Xi_{\Gamma(\gamma)}(\boldsymbol{\mu})
$$

fppn

$$
=1+\sum_{n \geq 1} \frac{1}{n !} \sum_{\left(\gamma_{1}, \ldots, \gamma_{n}\right) \in \mathcal{P}^{n}} \mu_{\gamma_{1}} \ldots \mu_{\gamma_{n}} \prod_{1 \leq i<j \leq n} \mathbb{1}_{\left\{\gamma_{i} \sim \gamma_{i}\right\}} \prod_{i=1}^{n} \mathbb{1}_{\left\{\gamma_{i} \not \gamma\right\}}
$$

then the series $|\Theta|_{\gamma}^{\Lambda}(\boldsymbol{\rho}),|\Pi|_{\gamma}^{\Lambda}(\boldsymbol{\rho})$ defined in (2.13), (2.14) are convergent and furthermore

bPia 
The form (2.18) of Dobrushin criterion shows more clearly the improvement brought by Theorem 2.2. In particular, since $\varphi_{\gamma}^{\mathrm{D}}(\boldsymbol{\mu}) \geq \varphi_{\gamma}^{\mathrm{FP}}(\boldsymbol{\mu})$, for any fixed $\gamma, \boldsymbol{\mu}$, the convergence radius $\boldsymbol{R}^{\mathrm{FP}}=\left\{\mu_{\gamma} / \varphi_{\gamma}^{\mathrm{FP}}(\boldsymbol{\mu})\right\}_{\gamma \in \mathcal{P}}$ given by theorem 2.2 is always greater than the the Dobrushin's crtiterion convergence radius $\boldsymbol{R}^{\mathrm{D}}=\left\{\mu_{\gamma} / \varphi_{\gamma}^{\mathrm{D}}(\boldsymbol{\mu})\right\}_{\gamma \in \mathcal{P}}$ (the $\boldsymbol{\mu}^{\prime}$ 's here are free parameters that can be adjusted to maximize the radii $\left.\boldsymbol{R}^{\mathrm{D}}, \boldsymbol{R}^{\mathrm{FP}}\right)$. The upper bound (2.25) is not explicitly given in reference [9]. It can be proven, however (see [2]), in a straightforward way from (2.24). Moreover it is easy to see (see again [2]) that (2.25) is an improvment of (2.21) for any $\boldsymbol{\rho} \leq \boldsymbol{R}^{\mathrm{D}}$.

The original statement by Dobrushin is obtained by substituting $\mu_{\gamma}+1=\mathrm{e}^{a_{\gamma}}$. In terms of these exponential weights the criterion is the existence of positive numbers $\boldsymbol{a}=\left\{a_{\gamma}\right\}_{\gamma \in \mathcal{P}}$ and $\boldsymbol{\rho}=\left\{\rho_{\gamma}\right\}_{\gamma \in \mathcal{P}}$ such that

r.dob.1

$$
\rho_{\gamma} \leq\left(\mathrm{e}^{\alpha_{\gamma}}-1\right) \exp \left(-\sum_{\tilde{\gamma} \in \Gamma(\gamma)} \alpha_{\tilde{\gamma}}\right)
$$

and the bound (2.21) becomes:

r.dob.2

$$
|\Theta|_{\gamma}^{\Lambda}(\boldsymbol{\rho}) \leq a_{\gamma}
$$

The earlier Kotecký-Preiss criterion [14] was the first to take the form (2.18), but with $\varphi_{\gamma}^{\mathrm{D}}$ replaced by the less efficient

$$
\varphi_{\gamma}^{\mathrm{KP}}=\exp \left\{\sum_{\tilde{\gamma} \in \Gamma(\gamma)} \mu_{\gamma}\right\} .
$$

The usual form of this condition, obtained upon substituting $\mu_{\gamma}=\rho_{\gamma} \mathrm{e}^{a_{\gamma}}$, is

$k p-a b s$

$$
\sum_{\tilde{\gamma} \in \Gamma(\gamma)} \rho_{\gamma} \mathrm{e}^{a_{\gamma}} \leq a_{\gamma} .
$$

\subsection{Subset gases: definition and convergence criteria}

Subset gases are particular types of polymer gases that appear in most of the uses of the cluster expansion in statistical mechanics. Their definition requires a countable set $\mathbb{V}$ that acts as an underlying "space". Polymers are then simply defined as the finite non empty subsets of $\mathbb{V}$, i.e.

$$
\mathcal{P}=\{\gamma \subset \mathbb{V}: 0<|\gamma|<\infty\}
$$

with non-empty intersection as incompatibility relation:

$$
\gamma \not \gamma^{\prime} \Longleftrightarrow \gamma \cap \gamma^{\prime} \neq \emptyset \text {. }
$$

Polymers can now be measured through its cardinality, so it makes sense about large and small polymers. The definition of the gas is completed by a family of activities $\boldsymbol{z}=\left\{z_{\gamma} \in \mathbb{C}\right\}_{\gamma \in \mathcal{P}}$.

In fact, the following discussion applies, more generally, to colored subset gases. These are systems in which polymers are endowed with some further attribute - the color - taken from some space $\mathcal{C}$. Formally, colored polymers are pairs $\gamma=(\underline{\gamma}, c)$ with $\underline{\gamma} \subset \mathbb{V}$ finite - the support of $\gamma$ - and $c \in \mathcal{C}$. Conspicuous examples are the "thick" contours of Pirogov-Sinai theory (see e.g. [25, Chapter II]) in which colors correspond to configurations on $\underline{\gamma}$. Colors do not play any role in the incompatibility relation, which remains as in (2.29) but involving supports, that is $\gamma \nsim \gamma^{\prime} \Longleftrightarrow \underline{\gamma} \cap \underline{\gamma}^{\prime} \neq \emptyset$. The following expressions remain valid for these more general contours if each $\gamma$ is identified with its support in geometrical statements (e.g. $\gamma \in \Lambda, x \in \gamma$, etc.)

For subset gases, the different objects of interest refer to parts of the underlying $\mathbb{V}$. Thus, subsets of $\mathbb{V}$ both are polymers and determine "finite-window" magnitudes. Because of their 
geometrical interpretation, subsets playing the latter role will be called "regions". The corresponding definitions - analogous to those for abstract polymers but with a slight and natural change in notation - are as follows. For a finite region $\Lambda \subset \mathbb{V}$ and contours $\gamma_{1}, \ldots \gamma_{n} \subset \Lambda$, the probability weights $\mathbb{P}_{\Lambda}$ are defined as in (2.1) with grand-canonical partition function

$$
\Xi_{\Lambda}(\boldsymbol{z})=1+\sum_{n \geq 1} \sum_{\substack{\left\{\gamma_{1}, \ldots, \gamma_{n}\right\} \subset \Lambda \\\left|\gamma_{i}\right| \geq 1, \gamma_{i} \cap \gamma_{j}=\emptyset}} z_{\gamma_{1}} \ldots z_{\gamma_{n}}
$$

With these partition functions, the pressure is defined also by (2.3) but for the correlations (2.4) we have the simplification that $\Gamma\left(\cup_{i=1}^{p} \gamma_{i}\right)$ is replaced by $\cup_{i=1}^{p} \gamma_{i}$. Therefore the reduced correlations take the form (2.6) for the partitions (2.30). The analogous of (2.9) are

$$
\begin{aligned}
\Theta_{x}^{\Lambda}(\boldsymbol{z}) & =\log \Xi_{\Lambda}(\boldsymbol{z})-\log \Xi_{\Lambda \backslash\{x\}}(\boldsymbol{z}) \\
& =\sum_{n=1}^{\infty} \frac{1}{n !} \sum_{\substack{\left(\gamma_{1}, \ldots, \gamma_{n}\right) \in \Lambda^{n} \\
\exists i: \gamma_{i} \ni x}} \phi^{T}\left(\gamma_{1}, \ldots, \gamma_{n}\right) z_{\gamma_{1}} \ldots z_{\gamma_{n}}
\end{aligned}
$$

for $x \in \Lambda$. The reconstruction formulas (2.10)-(2.11) hold with $\gamma_{i} \rightarrow x_{i}$. Note also that in the specific case of the subset gas the series $\Theta_{x}^{\Lambda}(\boldsymbol{z})$ and $\Pi_{\Lambda}^{\{x\}}(\boldsymbol{z})$ are in a very simple relation. Indeed,

$$
\Pi_{\Lambda}^{\{x\}}(\boldsymbol{z})=\frac{\partial}{\partial z_{\{x\}}} \log \Xi_{\Lambda}(\boldsymbol{z})=\frac{1}{\Xi_{\Lambda}(\boldsymbol{z})} \frac{\partial \Xi_{\Lambda}(\boldsymbol{z})}{\partial z_{\{x\}}}=\frac{\Xi_{\Lambda \backslash\{x\}}(\boldsymbol{z})}{\Xi_{\Lambda}(\boldsymbol{z})}=\exp \left\{-\Theta_{x}^{\Lambda}(\boldsymbol{z})\right\}
$$

whence, recalling (2.16) and (2.17), we get the identity

$$
|\Pi|_{\Lambda}^{\{x\}}(\boldsymbol{z})=\exp \left\{|\Theta|_{x}^{\Lambda}(\boldsymbol{\rho})\right\} .
$$

The convergence criteria for these polymers involve factorized weights of the form

fact

$$
\mu_{\gamma}=\prod_{x \in \gamma} \xi_{x} \equiv \xi^{\gamma}
$$

for some family $\boldsymbol{\xi}=\left\{\xi_{x}\right\}_{x \in \mathbb{V}}$ with each $\xi_{x}>0$. These single-site weights are always larger than one and are traditional parametrized as $\xi_{x}=\mathrm{e}^{a_{x}}$ with $a_{x}>0$. In the literature, the $a_{x}$ are invariably chosen independent of $x$ and equal to some common value $a$. With this choice

$$
\mu_{\gamma}=\mathrm{e}^{a|\gamma|} .
$$

In the sequel, however, we work with the more general choice (2.33). This introduces a minimal notational cost, but it reveals more clearly the essence of the different arguments and leads to more precise formulas.

The most widely used criterion is, in fact, Kotecký and Preiss' (2.28), written in the form

$$
\sup _{x \in \mathbb{V}} \sum_{\substack{\gamma \in \mathcal{P} \\ \gamma \ni x}} \rho_{\gamma} \mathrm{e}^{a|\gamma|} \leq a .
$$

Dobrushin criterion (Theorem 2.1) with the substitution $\mu_{\gamma}=\rho_{\gamma} e^{a|\gamma|}$ yields a strengthening of this condition that, however, has not been much used in practice. The earlier work of Gruber and Kunz contained already an even better condition (but upper bounds on correlations were divergent at the edge of convergence radius) . 
Theorem 2.3 [13. Let $a>0$ and $\boldsymbol{\rho}=\left\{\rho_{\gamma}\right\}_{\gamma \in \mathcal{P}}$ be collections of nonnegative numbers such that

$g k s-r 0$

$$
\sup _{x \in \mathbb{V}} \sum_{\substack{\gamma \in \mathcal{P} \\ x \in \gamma}} \rho_{\gamma} \mathrm{e}^{a|\gamma|}<e^{a}-1
$$

Then the functions (2.31) are analytic in the interior of the polydisc $\mathcal{D}_{\rho}$ and satisfy

$g k . b o 0$

$$
|\Theta|_{x}^{\Lambda}(\boldsymbol{\rho}) \leq \frac{1}{\mathrm{e}^{a}}\left[1+\sup _{x \in \mathbb{V}} \sum_{\substack{\gamma \in \mathcal{P} \\ x \in \gamma}} \rho_{\gamma} \mathrm{e}^{|\gamma|}\right]
$$

for all finite $\Lambda \subset \mathbb{V}$ and $x \in \Lambda$.

Theorem 2.2 yields a stronger result:

Theorem 2.4 [9]. Let $\boldsymbol{a}=\left\{a_{x}\right\}_{x \in \mathbb{V}}$ and $\boldsymbol{\rho}=\left\{\rho_{\gamma}\right\}_{\gamma \in \mathcal{P}}$ be collections of nonnegative numbers such that

$g k s-r$

$$
\sum_{\substack{\gamma \in \mathcal{P} \\ x \in \gamma}} \rho_{\gamma} \mathrm{e}^{\sum_{y \in \gamma} a_{y}} \leq e^{a_{x}}-1
$$

Then the functions (2.31) are analytic in the interior of the polydisc $\mathcal{D}_{\rho}$ and satisfy

$g k . b o$

$$
|\Theta|_{x}^{\Lambda}(\boldsymbol{\rho}) \leq a_{x}
$$

for all finite $\Lambda \subset \mathbb{V}$ and $x \in \Lambda$.

This result is obtained from (2.22)-(2.23) through some rather rough approximations. The argument is given in [9] for the choice (2.34), for completeness we show below the proof for general factorized weights. The bound (2.39) is the coarsest of a sequence of sharper bounds, as stated in Proposition 4.1 below.

Proof. We shall prove that condition (2.38) implies (2.22) for

fact-2

$$
\mu_{\gamma}=\mathrm{e}^{\sum_{x \in \gamma} a_{x}}
$$

With this choice the function (2.23) becomes

$$
\text { fp-series } \quad \varphi_{\gamma}^{\mathrm{FP}}(\boldsymbol{\mu})=1+\sum_{n=1}^{|\gamma|} \frac{1}{n !} \sum_{\left(\gamma_{1}, \ldots, \gamma_{n}\right) \in \mathcal{P}^{n}} \prod_{i=1}^{n} \rho_{\gamma_{i}} \mathrm{e}^{\sum_{y \in \gamma_{i}} a_{y}} \prod_{1 \leq i<j \leq n} \mathbb{1}_{\left\{\gamma_{i} \cap \gamma_{j}=\emptyset\right\}} \prod_{i=1}^{n} \mathbb{1}_{\left\{\gamma_{i} \cap \gamma \neq \emptyset\right\}}
$$

We now observe that a necessary condition to satisfy the indicator functions is the existence of different points $x_{i} \in \gamma_{i} \cap \gamma, i=1, \ldots, n$ that each $\gamma_{i}$ intersect $\gamma$ at a different point. Of course, the whole intersections must be disjoint but we only use the existence of some set of different points. This approximation is reasonably if the contours involved are small and very bad otherwise. In favorable case, this over-weighting of large contours may be masked by the smallness of the corresponding activities. Therefore,

$$
\varphi_{\gamma}^{\mathrm{FP}}(\boldsymbol{\mu}) \leq 1+\sum_{n=1}^{|\gamma|} \frac{1}{n !} \sum_{\substack{\left(x_{1}, \ldots, x_{n}\right) \in \gamma^{n} \\ x_{i} \neq x_{j}}} \prod_{i=1}^{n}\left[\sum_{\substack{\gamma \in \mathcal{P} \\ x_{i} \in \gamma}} \rho_{\gamma} \mathrm{e}^{\sum_{y \in \gamma} a_{y}}\right]
$$


Applying hypothesis (2.38) we obtain

$$
\begin{aligned}
\varphi_{\gamma}^{\mathrm{FP}}(\boldsymbol{\mu}) & \leq 1+\sum_{n=1}^{|\gamma|} \sum_{\left\{x_{1}, \ldots, x_{n}\right\} \subset \gamma} \prod_{i=1}^{n}\left[\mathrm{e}^{\left.a_{x_{i}}-1\right]}\right. \\
& =\prod_{x \in \gamma}\left[\left(\mathrm{e}^{a_{x}}-1\right)+1\right]=\mathrm{e}^{\sum_{x \in \gamma} a_{x}},
\end{aligned}
$$

and so $\rho_{\gamma} \varphi_{\gamma}^{\mathrm{FP}}(\boldsymbol{\mu}) \leq \rho_{\gamma} \mathrm{e}^{\sum_{x \in \gamma} a_{x}}=\mu_{\gamma}$ showing that (2.22) holds. Finally, bound (2.24) implies

$$
|\Pi|_{x}^{\Lambda}(\boldsymbol{\rho}) \leq \varphi_{\{x\}}^{\mathrm{FP}} \leq \mathrm{e}^{a_{x}}
$$

so using (2.32) we get the bound (2.39).

\subsection{Comments and overview of methods and results}

The preceding criteria suggested us a number of comments and questions that we answer in the sequel.

(C1) While Gruber-Kunz criterium improves the "naive" Dobrushin criterion obtained by the application of (2.18) to subset gases, the actual condition (2.38) is the natural analogue of (2.26) under the replacement $\sum_{\tilde{\gamma} \in \Gamma(\gamma)}$ by $\sum_{\tilde{\gamma} \ni x}$. This suggests that a correspondingly adaptated Dobrushin argument could lead to the same result. This is true, as discussed in Section 3, In fact such an argument proves the strongest criterion of Theorem 2.4.

(C2) The difference between convergence criteria of Theorems 2.4 and 2.3 looks small indeed. But the non-sharp inequality is clearly out of reach of the Gruber-Kunz treatment of Kirkwood-Salzburg equations, based on establishing strict contractions. Is this an inherent limitation of studies based on such equations? The answer is no, as we discuss in Section 4 The same equations processed in a different way do yield a proof of Theorem 2.4 .

(C3) Having proved that Dobrushin's and modified Gruber-Kunz approaches yield the same results for subset gases, it is natural to wonder whether this equivalence extends to general abstract polymers. The answer is yes, and we show this in Section 5.

There is an aspect, however, in which the modified GK-approach excels Dobrushin. The former leads to a whole sequence of successively better bounds for the ratios of partition functions, improving (2.27) or (2.39). See Propositions 4.1 and 5.1. A similar hierarchy of bounds appears in our general approach based on the Penrose identity [9].

The discussion that follows will clearly show the key point of contact between Dobrushin induction argument and Kirkwood-Salzburg equations. For the case of subset polymers, both rely on the site-addition identity

GKin 3

$$
\Xi_{Y \cup\{x\}}(\boldsymbol{z})=\Xi_{Y}(\boldsymbol{z})+\sum_{\substack{S \subset Y \\|S| \geq 0}} z_{\{x\} \cup S} \Xi_{Y \backslash S}(\boldsymbol{z})
$$

valid for any $Y \in \mathcal{P}$ and any $x \in \mathbb{V} \backslash Y$. This identity follows immediately from definition (2.30). In the general abstract setting there is an analogous polymer-addition identity consequence of $(2.2)$ :

$$
\Xi_{Z \cup \gamma_{0}}(\rho)=\Xi_{Z}(\boldsymbol{z})+z_{\gamma_{0}} \Xi_{Z \backslash \Gamma^{*}\left(\gamma_{0}\right)}(\boldsymbol{z})
$$


valid for any finite family $Z \subset \mathcal{P}$ and any polymer $\gamma_{0} \in \mathcal{P} \backslash Z$. Here $\Gamma^{*}\left(\gamma_{0}\right)$ denotes the punctured neighborhood of $\gamma_{0}$ :

$$
\Gamma^{*}\left(\gamma_{0}\right)=\Gamma\left(\gamma_{0}\right) \backslash\left\{\gamma_{0}\right\} .
$$

Identity (2.43), called "the fundamental identity" by Scott and Sokal ([24], section 3.1, formula (3.3)) is, as explained there, the key point of inductive proofs $\grave{a}$ la Dobrushin. The Kirkwood-Salzburg equations set up by Gruber and Kunz, on the other hand, follow from a rewriting of this fundamental identity. It is no surprise that both methods yield equivalent results.

\section{Induction method for the subset gas}

Let us start by proving Theorem 2.4 à la Dobrushin. As in the original Dobrushin argument, the proof is amazingly short. From identity (2.16) [consequence of the alternating-sign property] and the definition of $\Theta_{x}^{\Lambda}$ [first line of (2.31)] we see that the theorem is equivalent to the following proposition.

Proposition 3.1 Let $\boldsymbol{a}=\left\{a_{x}\right\}_{x \in \mathbb{V}}$ and $\boldsymbol{\rho}=\left\{\rho_{\gamma}\right\}_{\gamma \in \mathcal{P}}$ be collections of nonnegative numbers such that

$g k s-d o b$

$$
\sum_{\substack{\gamma \in \mathcal{P} \\ x \in \gamma}} \rho_{\gamma} \mathrm{e}^{\sum_{y \in \gamma} a_{y}} \leq \mathrm{e}^{a_{x}}-1
$$

then

$$
\frac{\Xi_{\Lambda \backslash\{x\}}(-\boldsymbol{\rho})}{\Xi_{\Lambda}(-\boldsymbol{\rho})} \leq \mathrm{e}^{a_{x}}
$$

for any finite $\Lambda \subset \mathbb{V}$ and any $x \in \Lambda$.

Proof. We proceed by induction on $|\Lambda|$. If $|\Lambda|=1, \Lambda=\{x\}$, hypothesis (3.1) implies that

$$
\rho_{\{x\}} \mathrm{e}^{a_{x}} \leq \sum_{\substack{\gamma \in \mathcal{P} \\ x \in \gamma}} \rho_{\gamma} \mathrm{e}^{\sum_{y \in \gamma} a_{y}} \leq \mathrm{e}^{a_{x}}-1 .
$$

Hence,

$$
\frac{\Xi_{\{x\} \backslash\{x\}}(-\boldsymbol{\rho})}{\Xi_{\{x\}}(-\boldsymbol{\rho})}=\frac{1}{\Xi_{\{x\}}(-\boldsymbol{\rho})}=\frac{1}{1-\rho_{x}} \leq \frac{1}{1-\left(1-\mathrm{e}^{-a_{x}}\right)}=\mathrm{e}^{a_{x}} .
$$

Assume (3.2) is true for $|\Lambda| \leq n$. Telescoping we conclude that

$$
\frac{\Xi_{\Lambda \backslash S}(-\boldsymbol{\rho})}{\Xi_{\Lambda}(-\boldsymbol{\rho})} \leq \mathrm{e}^{\sum_{y \in S} a_{y}}
$$

for any $S \subset \Lambda$. Take $x \notin \Lambda$. The site-addition identity (2.42) implies

$$
\frac{\Xi_{\Lambda \cup\{x\}}(-\boldsymbol{\rho})}{\Xi_{\Lambda}(-\boldsymbol{\rho})}=1-\sum_{S \subset \Lambda} \rho_{\{x\} \cup S} \frac{\Xi_{\Lambda \backslash S}(-\boldsymbol{\rho})}{\Xi_{\Lambda}(-\boldsymbol{\rho})}
$$

which by (3.3) yields

$$
\begin{aligned}
\frac{\Xi_{\Lambda \cup\{x\}}(-\boldsymbol{\rho})}{\Xi_{\Lambda}(-\boldsymbol{\rho})} & \geq 1-\sum_{S \subset \Lambda} \rho_{\{x\} \cup S} \mathrm{e}^{\sum_{y \in S} a_{y}} \\
& =1-\mathrm{e}^{-a_{x}} \sum_{\gamma \in \mathcal{P}: x \in \gamma} \rho_{\gamma} \mathrm{e}^{\sum_{y \in \gamma} a_{y}}
\end{aligned}
$$


Finally, using hypothesis (3.1),

$$
\frac{\Xi_{\Lambda \cup\{x\}}(-\boldsymbol{\rho})}{\Xi_{\Lambda}(-\boldsymbol{\rho})} \geq 1-\mathrm{e}^{-a_{x}}\left(\mathrm{e}^{a_{x}}-1\right)=\mathrm{e}^{-a_{x}} .
$$

Hence (3.2) holds for regions $\Lambda$ with $n+1$ sites.

We basically adapted the version of Miracle-Solé [15], who used identity (2.16) to simplify the original proof of Dobrushin which did not resort to such identity.

\section{Gruber-Kunz formalism for the subset gas}

In this section we revise the steps followed by Gruber and Kunz in [13] to arrive to their Theorem 2.3 and present the modifications needed to arrive to Theorem 2.4. In fact, the argument of Gruber and Kunz was presented in terms of a "gas of partitions" which basically corresponds to a gas of subsets with single-site fugacities equal to one. We have transcribed it to the framework of general subset gases.

\subsection{The proof by Gruber and Kunz}

Gruber and Kunz obtained their analyticity results by setting up linear equations for the reduced correlations $\bar{\phi}_{\Lambda}(\boldsymbol{z}, X)$ [defined by the subset version of (2.6)], involving a $\Lambda$-independent operator $K$. To find these equations Gruber and Kunz applied the so called "algebraic method" following closely Section 4.4. of [23. However, in the context of the subset gas, due to the fact that $\bar{\phi}_{\Lambda}(\boldsymbol{z}, X)$ are just ratios of partitions functions, their equations ([13] eq. (28) pag. 146) can be derived much more easily as follows. We start with the site-addition identity (2.42) written rather as a site-deletion identity, in the form

GKin4

$$
\Xi_{\Lambda \backslash X}(\boldsymbol{z})=\Xi_{\Lambda \backslash\left(X \backslash\left\{x_{1}\right\}\right)}(\boldsymbol{z})-\sum_{\substack{S \subset \Lambda \backslash X \\|S| \geq 0}} z_{\left\{x_{1}\right\} \cup S} \Xi_{\Lambda \backslash(X \cup S)}(\boldsymbol{z})
$$

valid for any finite $\Lambda \subset \mathbb{V}$ and any $x_{1} \in X \subset \Lambda$. Thus, upon dividing both sides by $\Xi_{\Lambda}(\boldsymbol{z})$,

GKin

$$
\bar{\phi}_{\Lambda}(\boldsymbol{z}, X)=\bar{\phi}_{\Lambda}\left(\boldsymbol{z}, X \backslash\left\{x_{1}\right\}\right)-\sum_{\substack{S \subset \Lambda \backslash X \\|S| \geq 0}} z_{\left\{x_{1}\right\} \cup S} \bar{\phi}_{\Lambda}(\boldsymbol{z}, X \cup S)
$$

These are what Gruber and Kunz call the Kirkwood-Salzburg equations for the gas of subsets. In these equations $x_{1}$ is some point of $X$ chosen once and for all, for instance as the smallest site of $X$ in some fixed enumeration of $\mathbb{V}$.

In order to write this in terms of a $\Lambda$-independent operator, it is necessary to include the restriction $X \subset \Lambda$ as a factor, so to extend the functions $\bar{\phi}_{\Lambda}(\boldsymbol{z}, X)$, defined only when $X \subset \Lambda$ to all $X \in \mathbb{V}$. Let us then define

chik

$$
\chi_{\Lambda}(X)=\mathbb{1}_{\{X \subset \Lambda\}}
$$

and denote

$$
\widetilde{\phi}_{\Lambda}(\boldsymbol{z}, X)=\chi_{\Lambda}(X) \bar{\phi}_{\Lambda}(\boldsymbol{z}, X)
$$


From (4.2) we obtain

tilphi

$$
\widetilde{\phi}_{\Lambda}(\boldsymbol{z}, X)=\left\{\begin{array}{c}
\chi_{\Lambda}(X) \widetilde{\phi}_{\Lambda}\left(\boldsymbol{z}, X \backslash\left\{x_{1}\right\}\right) \\
\chi_{\Lambda}(X)
\end{array}\right\}-\chi_{\Lambda}(X) \sum_{\substack{S \in \mathcal{P}^{*} \\
S \cap X=\emptyset}} \rho_{\left\{x_{1}\right\} \cup S} \widetilde{\phi}_{\Lambda}(\boldsymbol{z}, X \cup S)
$$

where $\mathcal{P}^{*}=\mathcal{P} \cup \emptyset$ and the upper line holds when $|X| \geq 2$ while the lower one when $|X|=1$. The latter includes the condition $\bar{\phi}_{\Lambda}(\boldsymbol{z}, \emptyset)=1$ which is better written as an inhomogenity of the linear system by introducing

$$
\alpha(X)=\mathbb{1}_{\{X \mid=1\}} .
$$

In this way we conclude that the function

$$
\widetilde{\phi}_{\Lambda}(\boldsymbol{z})(\cdot) \equiv \widetilde{\phi}_{\Lambda}(\boldsymbol{z}, \cdot): \mathcal{P}^{*} \longrightarrow \mathbb{C}
$$

satisfies the linear equation

$$
\widetilde{\phi}_{\Lambda}(\boldsymbol{z})=\chi_{\Lambda} \alpha+\chi_{\Lambda} K_{\boldsymbol{z}} \widetilde{\phi}_{\Lambda}(\boldsymbol{\rho})
$$

where $K_{\boldsymbol{z}}$ is the linear operator on the space of complex-valued functions on $\mathcal{P}$ defined by

Kro

$$
\left(K_{\boldsymbol{z}} f\right)(X)=\mathbb{1}_{\{|X| \geq 2\}} f\left(X \backslash\left\{x_{1}\right\}\right)-\sum_{\substack{S \in P^{*} \\ S \cap X=\emptyset}} z_{\left\{x_{1}\right\} \cup S} f(X \cup S) .
$$

At this point Gruber and Kunz resort to a contraction argument in Banach spaces. For this - having in mind weights of the form (2.33) - they associate to each family $\boldsymbol{\xi}=\left\{\xi_{x}\right\}_{x \in \mathbb{V}}$, with each $\xi_{x}>0$, the Banach space $\mathcal{B}_{\boldsymbol{\xi}}$ of complex functions defined on non empty finite subsets of $\mathbb{V}$ (i.e. on $\mathcal{P}$ ) with the norm

$$
\|f\|_{\xi}=\sup _{X \in \mathcal{P}} \frac{|f(X)|}{\xi^{X}} .
$$

where, for any $X \subset \mathbb{V}$, we abbreviate $\boldsymbol{\xi}^{X}=\prod_{x \in X} \xi_{x}$.

We have,

$k z k z$

$$
\begin{aligned}
\left|\left(K_{\boldsymbol{z}} f\right)(X)\right| & \leq \boldsymbol{\xi}^{X \backslash\left\{x_{1}\right\}}\|f\|_{\boldsymbol{\xi}}+\sum_{\substack{S \in P^{*} \\
S \cap X=\emptyset}}\left|z_{\left\{x_{1}\right\} \cup S}\right| \boldsymbol{\xi}^{X \cup S}\|f\|_{\boldsymbol{\xi}} \\
& =\boldsymbol{\xi}^{X}\|f\|_{\boldsymbol{\xi}} \frac{1}{\xi_{x_{1}}}\left[1+\sum_{\substack{\gamma \in \mathcal{P} \\
x \in \gamma}}\left|z_{\gamma}\right| \boldsymbol{\xi}^{\gamma} \cdot\right]
\end{aligned}
$$

Therefore $K_{\boldsymbol{z}}$ is a bounded operator in $\mathcal{B}_{\xi}$ with norm bounded by

normK

$$
\left\|K_{\boldsymbol{z}}\right\|_{\boldsymbol{\xi}} \leq \sup _{x \in \mathbb{V}} \frac{1}{\xi_{x}}\left[1+\sup _{x \in \mathbb{V}} \sum_{\substack{\gamma \in \mathcal{P} \\ x \in \gamma}}\left|z_{\gamma}\right| \xi^{|\gamma|}\right]
$$

If $\boldsymbol{z}$ is such that

norm

$$
\left\|K_{\boldsymbol{z}}\right\|_{\boldsymbol{\xi}}<1
$$

the equation (4.7) has a unique solution in the Banach space $\mathcal{B}_{\boldsymbol{\xi}}$ given by

$$
\widetilde{\phi}_{\Lambda}(\boldsymbol{z})=\left[1-\chi_{\Lambda} K_{\boldsymbol{z}}\right]^{-1} \chi_{\Lambda} \alpha
$$


By construction, this solution is analytic in $\boldsymbol{z}$ and furthermore

$b b b$

GKcondi

$$
\left\|\widetilde{\phi}_{\Lambda}(\boldsymbol{z})\right\|_{\boldsymbol{\xi}} \leq\left(1-\left\|K_{\boldsymbol{z}}\right\|_{\boldsymbol{\xi}}\right)^{-1}
$$

As the condition (4.12) is independent of $\Lambda$ the equation (4.13) makes sense in the limit $\Lambda \rightarrow \mathbb{V}$ and yields the convergence $\widetilde{\phi}_{\Lambda}(\boldsymbol{z}) \rightarrow \phi(\boldsymbol{z})$ where the latter is the unique solution of (4.13) without the factors $\chi_{\Lambda}$. Choosing $\xi_{x}=\mathrm{e}^{a}$ we see that the condition

$$
\frac{1}{\mathrm{e}^{a}}\left[1+\sup _{x \in \mathbb{V}} \sum_{\substack{\gamma \in \mathcal{P} \\ x \in \gamma}} \rho_{\gamma} \mathrm{e}^{|\gamma|}\right]<1
$$

implies the validity of all these properties for $z \in \mathcal{D}_{\rho}$ for all $\gamma \in \mathcal{P}$, plus analyticity in the interior of $\mathcal{D}_{\rho}$.

\subsection{Extended Gruber-Kunz criterion}

To extend the convergence region so to include equality in (4.15) and to improve bound (2.37) we have to abandon the precedent contraction strategy and find an alternative way to make sense of (4.13). In fact, this expression corresponds to the multivariate formal power series in $\boldsymbol{z}$

series

$$
\chi_{\Lambda} \sum_{n \geq 0}\left[\left(K_{\boldsymbol{z}}\right)^{n}\left(\chi_{\Lambda} \alpha\right)\right](X)
$$

Thus, by the reasons invoked above (4.15), it is enough to find a polydisc $\mathcal{D}_{\rho}$-independent of $\Lambda$ and $X$ - where all these series converge uniform and absolutely. A glimpse at the definition (4.8) of the operators $K_{\boldsymbol{z}}$ shows that if $\left|z_{\gamma}\right| \leq \rho_{\gamma}$ for all $\gamma \in \mathcal{P}$, each series (4.16) is term-by-term dominated by the series with positive terms

$$
\Phi_{\rho}(X)=\sum_{n \geq 0}\left[\left(K_{-\rho}\right)^{n} \alpha\right](X) .
$$

In particular, the reduced correlations satisfy

$$
\left|\bar{\phi}_{\Lambda}(z, X)\right| \leq \phi_{\Lambda}(-\boldsymbol{\rho}, X)=\frac{\Xi_{\Lambda \backslash X}(-\boldsymbol{\rho})}{\Xi_{\Lambda}(-\boldsymbol{\rho})} \leq \Phi_{\boldsymbol{\rho}}(X)
$$

for all finite $\Lambda$ and all $X \subset \Lambda, x \in \Lambda$ and $\left|z_{\gamma}\right| \leq \rho_{\gamma}$. To prove Theorem 2.4 we only need to find a $\Lambda$ - and $X$-independent family $\left\{\rho_{\gamma}>0\right\}_{\gamma \in \mathcal{P}}$ for which this series is finite. This is done in the following proposition which yields some further bonds.

Proposition 4.1 Let $\boldsymbol{\xi}=\left\{\xi_{x}\right\}_{x \in \mathbb{V}}$ and $\boldsymbol{\rho}=\left\{\rho_{\gamma}\right\}_{\gamma \in \mathcal{P}}$ be collections of nonnegative numbers such that

$$
\sum_{\substack{\gamma \in \mathcal{P} \\ x \in \gamma}} \rho_{\gamma} \boldsymbol{\xi}^{\gamma} \leq \xi_{x}-1
$$

for all $x \in \mathcal{P}$. Then the reduced correlations are analytic in the interior of the poly-disc $\mathcal{D}_{\rho}=$ $\left\{\left|z_{\gamma}\right| \leq \rho_{\gamma}: \gamma \subset \Lambda\right\}$ and satisfy the uniform bound

$$
\left|\frac{\Xi_{\Lambda \backslash X}(-\boldsymbol{z})}{\Xi_{\Lambda}(-\boldsymbol{z})}\right| \leq \boldsymbol{\xi}^{X}
$$


for all finite $\Lambda$, all $X \subset \Lambda$ and all $\mathbf{z} \in \mathcal{D}_{\rho}$. Furthermore, this bound can be systematically improved in the following way. Consider the operator $\mathbb{T}_{\rho}$ on functions $F$ on $\mathcal{P}$ defined by

$t t t$

seq.bo

iter

denote

$x K$

$K S E p$

$$
\left(\mathbb{T}_{\rho} F\right)(X) \equiv\left(\alpha+K_{-\rho} F\right)(X) .
$$

Then, for all $m \leq n$

$$
\left|\frac{\Xi_{\Lambda \backslash X}(-\boldsymbol{z})}{\Xi_{\Lambda}(-\boldsymbol{z})}\right| \leq \frac{\Xi_{\Lambda \backslash X}(-\boldsymbol{\rho})}{\Xi_{\Lambda}(-\boldsymbol{\rho})} \leq\left(\mathbb{T}_{\boldsymbol{\rho}}\right)^{m} \boldsymbol{\xi}^{X} \leq\left(\mathbb{T}_{\boldsymbol{\rho}}\right)^{n} \boldsymbol{\xi}^{X} \leq \boldsymbol{\xi}^{X}
$$

for all finite $\Lambda$, all $X \subset \Lambda$ and all $\mathbf{z} \in \mathcal{D}_{\rho}$.

\section{Proof.}

We start by observing that the positivity of the coefficients involved in the definition of $\mathbb{T}_{\boldsymbol{\rho}}$ implies that

$$
F(X) \leq G(X) \forall X \in \mathcal{P} \quad \Longleftrightarrow \quad\left(\mathbb{T}_{\rho} F\right)(X) \leq\left(\mathbb{T}_{\rho} G\right)(X) \forall X \in \mathcal{P} .
$$

Furthermore, for every non-negative function $\xi(X)$ and every positive integer $k$,

$$
\sum_{n=0}^{k}\left[\left(K_{-\boldsymbol{\rho}}\right)^{n} \alpha\right](X) \leq\left(\mathbb{T}_{\boldsymbol{\rho}}^{k+1} \xi\right)(X) .
$$

Claim 1: The series $\Phi_{\rho}(X)$ converge if and only if there exists a function $\xi(X): \mathcal{P}^{*} \longrightarrow[0, \infty)$ such that

$$
\left(\mathbb{T}_{\boldsymbol{\rho}} \xi\right)(X) \leq \xi(X) \quad \forall X \in \mathcal{P}
$$

Indeed, sufficiency follows from the fact that, by (4.24) and the monotonicity property (4.23),

$$
\Phi_{\rho}(X) \leq \lim _{k \rightarrow \infty}\left(\mathbb{T}_{\rho}^{k} \xi\right)(X) \leq \xi(X) .
$$

On the other hand, if the series $\Phi_{\rho}(X)$ converge, then (4.25) is satisfied - as equality - with $\xi(X)=\Phi_{\rho}(X)$.

Claim 2: If the family $\boldsymbol{\xi}=\left\{\xi_{x}\right\}_{x \in \mathbb{V}}$ satisfies (4.19), then the functions $\xi(X)=\boldsymbol{\xi}^{X}$ satisfy (4.25).

We need only to check (4.25) for $|X| \geq 2$. In this case

$$
\begin{aligned}
\left(\alpha+K_{-\rho} \xi\right)(X) & =\xi\left(X \backslash\left\{x_{1}\right\}\right)+\sum_{\substack{S \in P^{*} \\
S \cap X=\emptyset}} \rho_{\left\{x_{1}\right\} \cup S} \xi(X \cup S) \\
& =\boldsymbol{\xi}^{X \backslash\left\{x_{1}\right\}}\left[1+\sum_{\substack{\gamma \in \mathcal{P}^{*} \\
x_{1} \in \gamma}} \rho_{\gamma} \boldsymbol{\xi}^{\gamma}\right]
\end{aligned}
$$

As by (4.19) the last square bracket is less than $\xi_{x_{1}}$, the claim is proven.

Putting together the two claims we have proven the convergence of $\Phi_{\rho}$ whenever condition (4.19) is satisfied. As discussed above this yields analyticity in the interior of $\mathcal{D}_{\boldsymbol{\rho}}$. Successive applications of $\mathbb{T}_{\boldsymbol{\rho}}$ to both sides of (4.25) yield, by the monotonicity property (4.23) and the leftmost inequality in (4.26), the sequence of bounds

$$
\Phi_{\boldsymbol{\rho}}(X) \leq\left(\mathbb{T}_{\boldsymbol{\rho}}\right)^{m} \boldsymbol{\xi}^{X} \leq\left(\mathbb{T}_{\boldsymbol{\rho}}\right)^{n} \boldsymbol{\xi}^{X} \leq \boldsymbol{\xi}^{X} .
$$

Due to the bound (4.18) these inequalities prove (4.22). 


\section{Kirkwood-Salzburg formalism for the abstract polymer gas}

To conclude, we show how the approach of the previous section can be adapted to prove Dobrushin criterion (Theorem 2.1) through Kirkwood-Salzburg equations. As the treatment exactly parallels that for subset gases we shall only indicate the key expressions.

To derive the K-S equations we start from the polymer-addition identity $(2.43)$ - which is the same used by Dobrushin in his induction argument. Upon dividing by $\Lambda \supset Z \cup\left\{\gamma_{0}\right\}$ we obtain

absgk

$$
\frac{\Xi_{Z \cup \gamma_{0}}(\boldsymbol{z})}{\Xi_{\Lambda}(\boldsymbol{z})}=\frac{\Xi_{Z}(\boldsymbol{z})}{\Xi_{\Lambda}(\boldsymbol{z})}+z_{\gamma_{0}} \frac{\Xi_{Z \backslash \Gamma^{*}\left(\gamma_{0}\right)}(\boldsymbol{z})}{\Xi_{\Lambda}(\boldsymbol{z})}
$$

Choosing $Z=\Lambda \backslash X$ and writing $\Gamma_{\Lambda}^{*}\left(\gamma_{0}\right)=\Gamma^{*}\left(\gamma_{0}\right) \cap \Lambda$ we obtain that the reduced correlations (2.6) satisfy the equations

$$
\bar{\phi}_{\Lambda}(\boldsymbol{z}, X)=\bar{\phi}_{\Lambda}\left(\boldsymbol{z}, X \backslash \gamma_{0}\right)-z_{\gamma_{0}} \bar{\phi}_{\Lambda}\left(\boldsymbol{z}, X \cup \Gamma_{\Lambda}^{*}\left(\gamma_{0}\right)\right)
$$

These are the Kirkwood-Salzburg equations for the abstract polymer gas.

As in the previous section we introduce $\chi_{\Lambda}(X)=\mathbb{1}_{\{X \subset \Lambda\}}, \alpha(X)=\mathbb{1}_{\{|X|=1\}}$ and $\widetilde{\phi}_{\Lambda}(\boldsymbol{z}, X)=$ $\chi_{\Lambda}(X) \bar{\phi}_{\Lambda}(\boldsymbol{z}, X)$, so to write $(5.2)$ in the form

$K S E s$

$$
\widetilde{\phi}_{\Lambda}(\rho)=\chi_{\Lambda} \alpha+\chi_{\Lambda} K_{\rho}^{\Lambda} \widetilde{\phi}_{\Lambda}(\rho)
$$

with

opK

$$
\left(K_{z}^{\Lambda} f\right)(X)=\mathbb{1}_{\{|X| \geq 2\}} f\left(X \backslash \gamma_{0}\right)-z_{\gamma_{0}} f\left(X \cup \Gamma_{\Lambda}\left(\gamma_{0}\right)\right)
$$

where $\gamma_{0}$ is the first polymer in $X$ in some previously chosen enumeration. Note also that now $K_{z}^{\Lambda}$ depends also on $\Lambda$ since we recall that $\Gamma_{\Lambda}^{*}\left(\gamma_{0}\right)=\left\{\gamma \in \Lambda: \gamma \nsim \gamma_{0}\right\}$. The goal is to make sense of

line-abs

$$
\left[1-\chi_{\Lambda} K_{z}^{\Lambda}\right]^{-1} \chi_{\Lambda} \alpha=\chi_{\Lambda} \sum_{n \geq 0}\left[\left(K_{z}^{\Lambda}\right)^{n}\left(\chi_{\Lambda} \alpha\right)\right](X)
$$

simultaneously for all $\Lambda$.

We can now transcribe exactly the same steps as in the previous section with the notation

transc

$$
\xi^{X}=\prod_{\gamma \in X} \xi_{\gamma}
$$

In the original Gruber-Kunz approach the convergence of (5.5) is proven by showing that $K_{\mathbf{z}}$ is a contraction on the space $\mathbb{B}_{\boldsymbol{\xi}}$ of complex valued functions on polymers with norm $\|f\|_{\xi}$ defined as in (4.9). By a calculation completely analogous to (4.10) we obtain

normKs

$$
\left\|K_{\boldsymbol{z}}^{\Lambda}\right\|_{\boldsymbol{\xi}} \leq \sup _{\gamma_{0} \in \mathcal{P}}\left[\frac{1}{\xi_{\gamma_{0}}}\left[1+\left|z_{\gamma_{0}}\right| \prod_{\gamma \in \Gamma\left(\gamma_{0}\right)} \xi_{\gamma}\right]\right]
$$

With the substitution

subst

$$
\xi_{\gamma}=\mu_{\gamma}+1
$$

this yields the convergence condition

$$
\sup _{\gamma \in \mathcal{P}} \frac{\rho_{\gamma}}{\mu_{\gamma}} \prod_{\tilde{\gamma} \nsim \gamma}\left[1+\mu_{\tilde{\gamma}}\right]<1
$$


which is slightly weaker than Dobrushin condition (2.18)-(2.19).

To improve this condition we proceed as in the proof of Proposition 4.1 and focus rather on the convergence of the formal series defined by the right-hand-side of (5.5). A necessary and sufficient condition for this convergence is the existence of a function $\xi: \mathcal{P} \longrightarrow \mathbb{C}$ such that

KSEpas

$$
\left(\alpha+K_{-\rho} \xi\right)(X) \leq \xi(X)
$$

for every finite family $X \subset \mathcal{P}$. Here $K$ is the operator defined as in (5.4) but replacing $\Gamma_{\Lambda}\left(\gamma_{0}\right)$ by $\Gamma\left(\gamma_{0}\right)$.

opK 2

$$
\left(K_{\boldsymbol{z}}^{\Lambda} f\right)(X)=\mathbb{1}_{\{|X| \geq 2\}} f\left(X \backslash \gamma_{0}\right)-z_{\gamma_{0}} f\left(X \cup \Gamma_{\Lambda}\left(\gamma_{0}\right)\right)
$$

Assuming the factorization hypothesis (5.6) with $\xi_{g}>1$ we obtain, as in (4.27),

wewe

$$
\left(\alpha+K_{-\rho} \xi\right)(X) \leq \frac{\xi(X)}{\xi_{\gamma_{0}}}\left[1+\rho_{\gamma_{0}} \xi\left(\Gamma\left(\gamma_{0}\right)\right)\right]
$$

This bound, combined with condition (5.9) and the substitution (5.8) leads to the following proposition whose proof is a transcription of the proof of Proposition 4.1.

Proposition 5.1 Let $\boldsymbol{\xi}=\left\{\xi_{\gamma}\right\}_{\gamma \in \mathcal{P}}$ and $\boldsymbol{\rho}=\left\{\rho_{\gamma}\right\}_{\gamma \in \mathcal{P}}$ be collections of nonnegative numbers such that

dobpss

$$
\rho_{\gamma} \prod_{\tilde{\gamma} \in \Gamma(\gamma)} \xi_{\tilde{\gamma}} \leq \xi_{\gamma}-1
$$

for all $\gamma \in \mathcal{P}$. Then the reduced correlations are analytic in the interior of the poly-disc $\mathcal{D}_{\rho}=$ $\left\{\left|z_{\gamma}\right| \leq \rho_{\gamma}: \gamma \subset \Lambda\right\}$ and satisfy the uniform bound

uniffp

$$
\left|\frac{\Xi_{\Lambda \backslash X}(-z)}{\Xi_{\Lambda}(-\boldsymbol{z})}\right| \leq \boldsymbol{\xi}^{X}
$$

for all finite $\Lambda$, all $X \subset \Lambda$ and all $\mathbf{z} \in \mathcal{D}_{\rho}$. Furthermore, this bound can be systematically improved in the following way. Consider the operator $\mathbb{T}_{\rho}$ on functions $F$ on $\mathcal{P}$ defined by

$t t t f p$

$$
\left(\mathbb{T}_{\rho} F\right)(X) \equiv\left(\alpha+K_{-\rho} F\right)(X) .
$$

Then, for all $m \leq n$

seq.bofp

$$
\left|\frac{\Xi_{\Lambda \backslash X}(-\boldsymbol{z})}{\Xi_{\Lambda}(-\boldsymbol{z})}\right| \leq \frac{\Xi_{\Lambda \backslash X}(-\boldsymbol{\rho})}{\Xi_{\Lambda}(-\boldsymbol{\rho})} \leq\left(\mathbb{T}_{\boldsymbol{\rho}}\right)^{m} \boldsymbol{\xi}^{X} \leq\left(\mathbb{T}_{\boldsymbol{\rho}}\right)^{n} \boldsymbol{\xi}^{X} \leq \boldsymbol{\xi}^{X}
$$

for all finite $\Lambda$, all $X \subset \Lambda$ and all $\mathbf{z} \in \mathcal{D}_{\rho}$.

Note that (5.12) becomes Dobrushin's criterion (2.18) by substituting $\xi_{\gamma}=1+\mu_{\gamma}$.

\section{Conclusion}

The precedent arguments show that inductive DKP arguments and the use of KS equations are basically two alternative ways of exploiting the site-addition or polymer-addition identities (2.42) and (2.43). As the Kirkwood-Salzburg equations are exact relations between reduced correlations, they potentially include all the information needed to obtain successive improvements. 
In fact, our analysis show where to aim: better bounds require better choices of functions $\xi(X)$ satisfying (5.9). Such functions must not, therefore, be of the factorized form (5.6). Expression (5.11) implies the necessary condition

$$
1+\rho_{\gamma_{0}} \xi\left(\Gamma\left(\gamma_{0}\right)\right) \leq \xi_{\gamma_{0}}
$$

for all $\gamma_{0} \in \mathcal{P}$. In fact, the condition found in [9] is exactly of this form with $\xi(X)=\Xi_{X}$. We have been unable, however to prove the validity of (5.9) for such a function $\xi$ for arbitrary $X$.

\section{Acknowledgments}

This work has been partially supported by Conselho Nacional de Desenvolvimento Científico e Tecnológico (CNPq), CAPES (Coordenação de Aperfeiçoamento de Pessoal de Nível Superior, Brasil) and FAPEMIG (Fundacão de Amparo à Pesquisa do Estado de Minas Gerais). RB thanks the hospitality of Laboratoire de Mathématiques Raphaël Salem in the Université de Rouen during his PhD work. RF and AP thank The Newton Institute for the generous support during the Combinatorics and Statistical Mechanics programme.

\section{References}

[1] Bissacot, R. (2009): Técnicas para convergência da Expansão do Gás de Polímeros e uma aplicação ao Método Probabilístico. Thesis. Universidade Federal de Minas Gerais.

[2] Bissacot, R.; Fernández, R.; Procacci, A.; Scoppola, B.: "An Improvement of the Lovász Local Lemma via Cluster Expansion", Preprint, arXiv:0910.1824 [math.CO].

[3] Bovier, A. and Zahradnik, M. (2000): "A simple inductive approach to the problem of convergence of cluster expansions of polymer models". J. Statist. Phys. 100, 765-78.

[4] Brydges, D. C. (1984): "A short cluster in cluster expansions". In Critical Phenomena, Random Systems, Gauge Theories, Osterwalder, K. and Stora, R. (eds.), Elsevier, 129-83.

[5] Brydges, D. C., Martin, Ph. A. (1999): "Coulomb Systems at Low Density: A Review". J. Statist. Phys. 96, 1163-1330.

[6] Cammarota, C. (1982): "Decay of correlations for infinite range interactions in unbounded spin systems". Comm. Math. Phys. 85, 517-28.

[7] Dobrushin, R. L. (1996): "Estimates of semiinvariants for the Ising model at low temperatures". Topics in Statistics and Theoretical Physics, Amer. Math. Soc. Transl. (2), 177, 59-81.

[8] Dobrushin, R. L. (1996a): "Perturbation methods of the theory of Gibbsian fields". In Ecole d'Eté de Probabilités de Saint-Flour XXIV - 1994, Springer-Verlag (Lecture Notes in Mathematics 1648), Berlin-Heidelberg-New York, 1-66.

[9] Fernandez, R.; Procacci A.(2007): "Cluster expansion for abstract polymer models.New bounds from an old approach " Comm. in Math. Phys. 274, n.1, 123-140.

[10] Fernandez, R.; Procacci A. (2008): "Regions without complex zeros for chromatic polynomials on graphs with bounded degree". Comb. Prob. Comp., 17, 225-238. 
[11] Fernandez, R.; Procacci A.; Scoppola, B. (2007): "The analyticity region of the hard sphere gas. Improved bounds". J. of Statist. Phys., 128, n.5 1139-1143.

[12] Jackson, B.; Procacci, A.; Sokal A. D.: "Complex zero-free regions at large $|q|$ for multivariate Tutte polynomials (alias Potts-model partition functions) with general complex edge weights", preprint arXiv:0810.4703v2 [math.CO].

[13] Gruber, C.; Kunz, H. (1971): "General properties of polymer systems". Comm. Math. Phys. 22, 133-61.

[14] Kotecký, R.; Preiss, D. (1986): "Cluster expansion for abstract polymer models". Commun. Math. Phys., 103, 491-498.

[15] Miracle-Solé, S. (2000): "On the convergence of cluster expansions". Physica A 279, 244-9.

[16] Nardi, F. R.; Olivieri, E.; Zahradnik M. (1999): "On the Ising model with strongly anisotropic external field". J. Stat. Phys. 97, 87-144.

[17] Penrose, O. (1967): "Convergence of fugacity expansions for classical systems". In Statistical mechanics: foundations and applications, A. Bak (ed.), Benjamin, New York.

[18] Poghosyan, S.; Ueltschi, D. (2009): "Abstract cluster expansion with applications to statistical mechanical systems". J. Math. Phys. 50, 053509.

[19] Procacci A. (2009): "Erratum and Addendum: Abstract polymer models with general pair interactions". J. of Statist. Phys. 135, 779-786.

[20] Procacci A. (2007): "Abstract polymer models with general pair interactions". J. of Statist. Phys. 129, 171-188.

[21] Procacci, A.; de Lima, B. N. B.; Scoppola, B. (1998): "A remark on high temperature polymer expansion for lattice systems with infinite range pair interactions". Lett. Math. Phys. 45, no. 4, 303-322.

[22] Procacci A.; Scoppola, B. (1999): "Polymer gas approach to N-body lattice systems". J. of Statist. Phys., 96, 49-68.

[23] Ruelle, D. (1969). "Statistical mechanics: Rigorous results". W. A. Benjamin, Inc., New York-Amsterdam.

[24] Scott, A.; Sokal, A. D. (2005): "The repulsive lattice gas, the independent-set polynomial, and the Lovász local lemma". J. Statist. Phys. 118, 1151-261.

[25] Sinai, Ya. G. (1982). "Theory of Phase Transitions: Rigorous Results". Pergamon Press, Oxford-New York-etc.

[26] Sokal, A. D. (2001): "Bounds on the complex zeros of (di)chromatic polynomials and Pottsmodel partition functions". Combin. Probab. Comput. 10, 41-77.

[27] Ueltschi, D. (2004): "Cluster expansions and correlation functions". Mosc. Math. J. 4, $511-22,536$. 